\title{
Computational Curriculum for MatSE Undergraduates and the Influence on Senior Classes
}

\section{Xiao Zhang, University of Illinois, Urbana-Champaign}

Xiao Zhang is $\mathrm{PhD}$ student in the Department of Mechanical Science and Engineering in the University of Illinois, Urbana-Champaign and he is currently working in the Schleife's group in the Materials science and Engineering department. His research focus is simulating optical properties of materials using DFT based method and perturbation theory (GW/BSE). He is the computational teaching assistant of the MatSE SIIP team for the academic year of 2017-2018.

\section{Prof. Andre Schleife, University of Illinois at Urbana-Champaign}

André Schleife is a Blue Waters Assistant Professor in the Department of Materials Science and Engineering at the University of Illinois at Urbana-Champaign. He obtained his Diploma and Ph.D. at FriedrichSchiller-University in Jena, Germany for his theoretical work on transparent conducting oxides. Before he started at UIUC he worked as a Postdoctoral Researcher at Lawrence Livermore National Laboratory on a project that aimed at a description of non-adiabatic electron ion dynamics. His research revolves around excited electronic states and their dynamics in various materials using accurate computational methods and making use of modern super computers in order to understand, for instance, how light is absorbed in photo-voltaic materials.

\section{Prof. Andrew Ferguson, University of Illinois, Urbana-Champaign}

Andrew Ferguson is Associate Professor of Materials Science and Engineering, and Chemical and Biomolecular Engineering, and an Affiliated Associate Professor of Physics, and Computational Science and Engineering at the University of Illinois at Urbana-Champaign. He received an M.Eng. in Chemical Engineering from Imperial College London in 2005, and a Ph.D. in Chemical and Biological Engineering from Princeton University in 2010. From 2010 to 2012 he was a Postdoctoral Fellow of the Ragon Institute of MGH, MIT, and Harvard in the Department of Chemical Engineering at MIT. He commenced his appointment at UIUC in August 2012. His research interests lie in materials science, molecular simulation, and machine learning, with particular directions in accelerated sampling using deep and manifold learning, and the design of antiviral vaccines, antimicrobial peptides, and self-assembling colloids and peptides. $\mathrm{He}$ is the recipient of a 2017 UIUC College of Engineering Dean's Award for Excellence in Research, 2016 AIChE CoMSEF Young Investigator Award for Modeling \& Simulation, 2015 ACS OpenEye Outstanding Junior Faculty Award, 2014 NSF CAREER Award, 2014 ACS PRF Doctoral New Investigator, and was named the Institution of Chemical Engineers North America 2013 Young Chemical Engineer of the Year.

\section{Dr. Pascal Bellon, University of Illinois, Urbana-Champaign}

After earning a PhD in Materials Science from University of Paris 6, France, Pascal Bellon worked during 7 years at CEA-Saclay, France, before joining the Department of Materials Science and Engineering at the University of Illinois at Urbana-Champaign as a tenure-track Assistant Professor in 1996, where he was promoted to the ranks of Associate Professor in 2002 and Full Professor in 2009. He received an NSF career award in 1998 and awards from the Academy for Excellence in Engineering Education from the University of Illinois in 1998, 1999 and 2000. He received the Don Burnett teaching award in 2000, the Accenture Engineering council award for Excellence in Advising in 2007 and the Stanley Pierce award in 2009. In 2012 Pascal Bellon was named a Racheff faculty scholar, and in 2016 he was inducted as the Donald W. Hamer Professor in Materials Science and Engineering.

\section{Prof. Timothy Bretl, University of Illinois at Urbana-Champaign}

Timothy Bretl is an Associate Professor of Aerospace Engineering at the University of Illinois at UrbanaChampaign. He received his B.S. in Engineering and B.A. in Mathematics from Swarthmore College in 1999, and his M.S. in 2000 and Ph.D. in 2005 both in Aeronautics and Astronautics from Stanford 
University. Subsequently, he was a Postdoctoral Fellow in the Department of Computer Science, also at Stanford University. He has been with the Department of Aerospace Engineering at Illinois since 2006, where he now serves as Associate Head for Undergraduate Programs. He holds an affiliate appointment in the Coordinated Science Laboratory, where he leads a research group that works on a diverse set of projects (http://bretl.csl.illinois.edu/). Dr. Bretl received the National Science Foundation Early Career Development Award in 2010. He has also received numerous awards for undergraduate teaching in the area of dynamics and control, including all three teaching awards given by the College of Engineering at Illinois (the Rose Award for Teaching Excellence, the Everitt Award for Teaching Excellence, and the Collins Award for Innovative Teaching).

\section{Dr. Geoffrey L Herman, University of Illinois, Urbana-Champaign}

Dr. Geoffrey L. Herman is a teaching assistant professor with the Deprartment of Computer Science at the University of Illinois at Urbana-Champaign. He also has a courtesy appointment as a research assistant professor with the Department of Curriculum \& Instruction. He earned his Ph.D. in Electrical and Computer Engineering from the University of Illinois at Urbana-Champaign as a Mavis Future Faculty Fellow and conducted postdoctoral research with Ruth Streveler in the School of Engineering Education at Purdue University. His research interests include creating systems for sustainable improvement in engineering education, conceptual change and development in engineering students, and change in faculty beliefs about teaching and learning. He serves as the Publications Chair for the ASEE Educational Research and Methods Division.

Prof. Jessica A. Krogstad, Department of Materials Science and Engineering, University of Illinois at UrbanaChampaign

Jessica A. Krogstad is an assistant professor in the Department of Material Science and Engineering at the University of Illinois, Urbana-Champaign. She received her $\mathrm{PhD}$ in Materials at the University of California, Santa Barbara in 2012. Between 2012 and 2014, she held a postdoctoral appointment in the Department of Mechanical Engineering at Johns Hopkins University. Her current research explores the interplay between phase or morphological evolution and material functionality in structural materials under extreme conditions.

Prof. Robert Maass, Department of Materials Science and Engineering, University of Illinois at UrbanaChampaign

Robert Maass received a triple diploma in Materials Science and Engineering from the Institut National Polytechnique de Lorraine (INPL-EEIGM, France), Luleå Technical University (Sweden) and Saarland University (Germany) in 2005. In 2009, he obtained his PhD from the Materials Science Department at the École Polytechnique Fédérale de Lausanne (EPFL) in Switzerland. During his doctoral work, Robert designed and built an in-situ micro-compression set-up that he used to study small-scale plasticity with time-resolved Laue diffraction at the Swiss Light Source. From 2009-2011 he worked as a postdoctoral researcher at the Swiss Federal Institute of Technology (ETH Zurich) on plasticity of metallic glasses. Subsequently, he joined the California Institute of Technology as an Alexander von Humboldt postdoctoral scholar to continue his research on plasticity of metals. After working as a specialist management consultant for metals at McKinsey \& Co., he transferred to the University of Göttingen as a junior research group leader. He joined the faculty of the University of Illinois at Urbana-Champaign as Assistant Professor of Materials Science and Engineering in 2015.

\section{Dr. Cecilia Leal, University of Illinois at Urbana-Champaign}

Cecília Leal is an Assistant Professor in the Department of Materials Science and Engineering and the Frederick Seitz Materials Research Laboratory at the University of Illinois, Urbana-Champaign since 2012. She graduated in Industrial Chemistry from Coimbra University in Portugal and received her Ph.D. in physical chemistry from Lund University, supervised by Prof. Wennerström. After working for a year in the Norwegian Radium Hospital, she joined Prof. Safinya's Lab at the University of California in Santa 
Barbara as a postdoctoral fellow. Her research interests focus on the characterization and functionalization of lipid materials for cellular delivery. She is the recipient of a number of distinctions including the National Science Foundation CAREER award and the NIH New innovator award.

\section{Prof. Dallas R Trinkle, University of Illinois, Urbana-Champaign}

Dallas R. Trinkle is an associate professor in Materials Science and Engineering at Univ. Illinois, UrbanaChampaign. He received his Ph.D. in Physics from Ohio State University in 2003. Following his time as a National Research Council postdoctoral researcher at the Air Force Research Laboratory, he joined the faculty of the Department of Materials Science and Engineering at Univ. Illinois, Urbana-Champaign in 2006. He was a TMS Young Leader International Scholar in 2008, received the NSF/CAREER award in 2009, the Xerox Award for Faculty Research at Illinois in 2011, the AIME Robert Lansing Hardy Award in 2014, co-chaired the 2011 Physical Metallurgy Gordon Research conference, and became a Willett Faculty Scholar at Illinois in 2015. His research focuses on defects in materials using density-functional theory, and novel techniques to understand problems in mechanical behavior and transport.

\section{Prof. Matthew West, University of Illinois, Urbana-Champaign}

Matthew West is an Associate Professor in the Department of Mechanical Science and Engineering at the University of Illinois at Urbana-Champaign. Prior to joining Illinois he was on the faculties of the Department of Aeronautics and Astronautics at Stanford University and the Department of Mathematics at the University of California, Davis. Prof. West holds a Ph.D. in Control and Dynamical Systems from the California Institute of Technology and a B.Sc. in Pure and Applied Mathematics from the University of Western Australia. His research is in the field of scientific computing and numerical analysis, where he works on computational algorithms for simulating complex stochastic systems such as atmospheric aerosols and feedback control. Prof. West is the recipient of the NSF CAREER award and is a University of Illinois Distinguished Teacher-Scholar and College of Engineering Education Innovation Fellow. 


\title{
Computational Curriculum for MatSE Undergraduates: Three-year impact and influence on senior computational classes
}

\author{
Xiao Zhang ${ }^{1}$, André Schleife ${ }^{2}$, Andrew Ferguson ${ }^{2}$, Pascal Bellon ${ }^{2}$, Timothy \\ Brett $^{3}$, Geoffrey L Herman ${ }^{4}$, Jessica A Krogstad ${ }^{2}$, Robert Maass², Cecilia Leal ${ }^{2}$, \\ Dallas R Trinkle ${ }^{2}$, Matthew West ${ }^{2}$, and Jian Ku Shang ${ }^{2}$ \\ ${ }^{1}$ Department of Mechanical Science and Engineering, University of Illinois at \\ Urbana-Champaign, Urbana, IL, USA \\ ${ }^{2}$ Department of Materials Science and Engineering, University of Illinois at \\ Urbana-Champaign, Urbana, IL, USA \\ ${ }^{3}$ Department of Aerospace Engineering, University of Illinois at \\ Urbana-Champaign, Urbana, IL, USA \\ ${ }^{4}$ Department of Computer Science, University of Illinois at Urbana-Champaign, \\ Urbana, IL, USA
}

\section{Abstract}

Computational materials simulation and modeling has emerged as a cornerstone of materials science research. At the University of Illinois at Urbana-Champaign, our faculty team in the Department of Materials Science and Engineering (MatSE), as part of the Strategic Instructional Initiatives Program (SIIP) of the university, have integrated comprehensive computational modules into multiple MatSE undergraduate classes and have created a collaborative teaching environment to iteratively improve these modules. Each year, a dedicated teaching assistant has been involved to communicate between faculty members, ensure the quality of the computational modules, assist in their delivery, and offer supplementary computational office hours. After three years of effort, we have now established a stable environment for computational education within MatSE undergraduate classes. The students initially involved in the program are now approaching their senior years. In this paper, we present the recent progress of our computational curriculum and focus on the influence of the program on the performance of students in senior computational modeling classes and senior classes with computational modules.

\section{Introduction}

Computational modeling of materials properties has grown increasingly important in both academia and industry.[1] Survey data from 2009 shows that the an average of $50 \%$ of new hires are expected to have computational training experience. [1] Due to the well-known need of computational skills, it is vital to provide computational training to undergraduate students in materials 
science.[1, 2] A recent study by Vieira and others[3] shows that integrating computational contents to a thermodynamics class not only increases students' understanding of the class materials, but also increases their confidence of doing computational simulations overall.

In the Department of Materials Science and Engineering at the University of Illinois at UrbanaChampaign, our faculty team has developed a program to integrate computational modules into existing core classes of the undergraduate curriculum. With such a scheme, we provide computational training without adding additional classes to the curriculum.[4, 5] In this paper, we report recent progress of this program, we analyze feedback obtained from the students, and we discuss the impact of the program on senior-level elective classes that focus entirely on computational skills.

\section{Curricular Reform and Computational Modules}

The Strategic Instructional Initiatives Program (SIIP) is inspired by Henderson et al. [6-9] and discussed in Refs. $[4,5,10]$ The SIIP program aims to establish a collaborative teaching environment to enhance instruction, and is aimed primarily at core classes with large enrollments. In this context, we created a collaborative platform in the MatSE department, targeting a series of classes ranging from 200-level sophomore classes that are a required part of the curriculum, to 400-level senior electives. In all classes, two to three computational modules are implemented. To ensure the quality of the module, a dedicated graduate student teaching assistant is trained in educational delivery of computational materials science content[11] through direct mentoring from the faculty team and attendance at the Summer School for Integrated Computational Materials Education (https: / / i cmed.engin.umich.edu/) to provide support for development and deployment of the modules. The modules are collaboratively and sustainably developed within a faculty community of practice anchored by regular meetings, online repositories, and the shared teaching assistant. The computational modules are incorporated in the classes through dedicated homework problem problems and discussion of computational material in course lectures. The teaching assistant holds dedicated "computational office hours" in addition to any regularly scheduled office hours for the course to help students understand and use computational software packages, and support their use of these packages to solve the computational homework problems. The computational content is designed to directly engage with the class material, and the skills and training needed to use computational software is provided by the faculty member and teaching assistant. In instances where the class instructor may lack expertise in the computational software, they are provided training by other faculty possessing these expertise, and the teaching assistant - who has been previously trained - may take the lead in delivering the material.

The list of classes and faculty members involved in the program can be found in Table 1 and Table 2, respectively. There are a total of nine faculty members currently involved in the program. We only listed the faculty members who participated in the SIIP program in Spring 2017 and Fall 2017 as the information about previous years can be found in Ref. [5].

After three years of effort, the progress of the program has reached a steady state. This year, the computational modules of the classes involved remain the same as described in the previous 
Table 1: Classes directly involved in the SIIP program and senior level computational classes (marked by $\dagger$ ) that are potentially affected by the effectiveness of SIIP program.

\begin{tabular}{c|l|c|c} 
Number & Name & Level & Type \\
\hline 201 & Phase and Phase Relations & Sophomore & Required \\
206 & Mechanics for MatSE & Sophomore & Required \\
304 & Electronic Properties of Materials & Junior & Semi-required \\
401 & Thermodynamics of Materials & Required \\
402 & Kinetic Processes in Materials & Junior & Required \\
406 & Thermal and Mechanical Behavior of Materials & Junior & Required \\
440 & Mechanical Behavior of Materials & Junior/Senior & Semi-required \\
$404 \dagger$ & Laboratory Studies in MatSE: Computational MatSE & Senior & Elective \\
$485 \dagger$ & Atomic Scale Simulations & Senior & Elective
\end{tabular}

Table 2: SIIP classes and the corresponding faculty members in Spring 2017 and Fall 2017. Marks of $\dagger$ indicate that computational modules are used in the class.

\begin{tabular}{c|c|c} 
Class & Spring 2017 & Fall 2017 \\
\hline 201 & Shang $\dagger$ & Leal $\dagger$ \\
206 & Krogstad $\dagger$ & Shang \\
304 & Schleife $\dagger$ & \\
401 & & Dillon $\dagger$ \\
402 & Bellon $\dagger$ & \\
406 & & Maass $\dagger$ \\
440 & & Krogstad $\dagger$ \\
404 & & Ferguson $\dagger$
\end{tabular}

report.[5] The computational software packages employed are:

- Quantum Espresso[12] for density functional theory (DFT)

- LAMMPS[13] for molecular dynamics (MD)

- OVITO[14] for atomic visualization

- OOF2[15] for finite element method (FEM)

- Thermo-Calc[16] (CALPHAD) for phase diagrams

- MATLAB[17] for numerical computing

The usage of these different packages in the classes are listed in Table 3. Below, we present the survey data obtained in the classes that are taught in Fall 2017. As shown in Table 3, even excluding MSE 404, which is the computation-focused senior elective, the Fall 2017 classes still cover all modules that we integrated into the curriculum.

As the program matures, it is important to obtain frequent student feedback and to analyze its 
Table 3: Computational methods integrated in SIIP classes. Classes marked by * are classes where computational modules are used in Fall 2017 and are the main source of survey data in this paper.

\begin{tabular}{c|c|c|c|c|c} 
Course & DFT & MD & FEM & CALPHAD & MATLAB \\
$201 *$ & $\mathrm{X}$ & & & $\mathrm{X}$ & \\
206 & & & $\mathrm{X}$ & & $\mathrm{X}$ \\
304 & $\mathrm{X}$ & & & & \\
401 & & $\mathrm{X}$ & & $\mathrm{X}$ & \\
402 & & $\mathrm{X}$ & & & $\mathrm{X}$ \\
$406 *$ & & $\mathrm{X}$ & $\mathrm{X}$ & & \\
$440 *$ & & & $\mathrm{X}$ & & $\mathrm{X}$ \\
$404 *$ & $\mathrm{X}$ & $\mathrm{X}$ & $\mathrm{X}$ & $\mathrm{X}$ & $\mathrm{X}$
\end{tabular}

efficacy and identify areas for improvement. In this paper, we discuss the surveys that we conducted to shed light on aspects including the students' familiarity and confidence towards the computational tools, their attitude towards the importance of computational skills, and towards their satisfaction with the curriculum. These surveys are conducted before students enter a class and after they finish a class.

The rest of the paper is structured as follows. In section 4, we discuss the impact of the SIIP program on the MSE students' attitude towards computational skills. In section 5, we discuss the impact of the SIIP program on the senior level classes that focus completely on computational materials science. We provide further discussion of the importance of more effective feedback in section 6 . In section 7, we present our conclusions and outlook.

\section{Impact of the program within core undergraduate classes}

In this section, we analyze survey data collected from the core undergraduate SIIP classes to evaluate the effectiveness of the SIIP program, and to identify areas for future improvement.

\subsection{Earlier exposure to computation}

In this section, we show that student feedback indicates a desire for earlier exposure to computational tools. A survey question in the first class that involves computational modules MSE 201 relates to the desired time to learn computational skills:

- "When do you think it is the best time to learn about computational tools for materials science and engineering?"

Figure 1 shows the results of the survey. It shows that after taking the first class with computational modules in it, student attitude towards the desired time of learning computational skills shifted to earlier in their curriculum. Before taking MSE 201, students' exposure to computational materials science is very limited. Although students have taken CS 101 as a required class, 


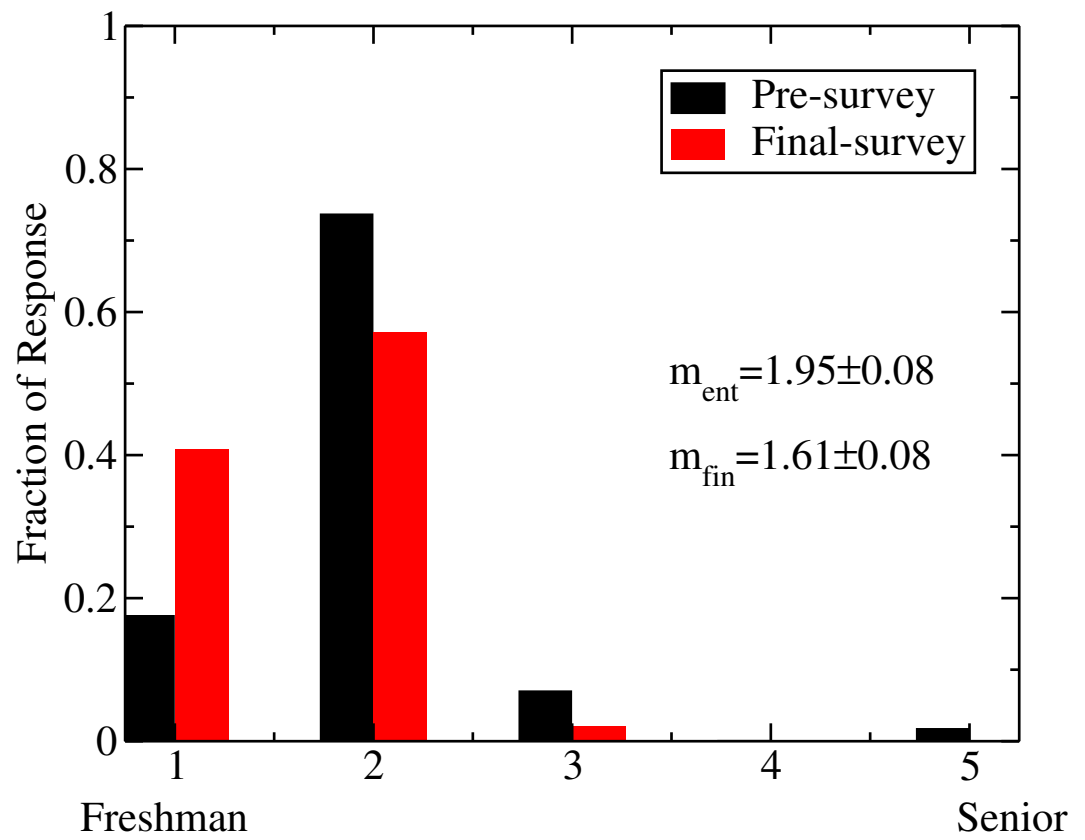

Figure 1: The entry-survey and final-survey result of the desired time of learning computational skills in MSE 201 ("When do you think it is the best time to learn about computational tools for materials science and engineering?"). The sample size is 57 in the entry and 49 in the final survey. $m_{\text {ent }}$ and $m_{\text {fin }}$ denote the mean value of the entry survey and final survey.

no connections to materials sciences have been made as they have never had a formal MatSE class with computational modules. With this background, the majority of students believes that learning computational skills in the second year is optimal. However, after completing MSE 201, the average of the time which student think is the best to learn computational skills shifted from $1.95 \pm 0.08$ to $1.61 \pm 0.08$. It can also be seen from Figure 1 that after finishing MSE 201, the proportion of students who have chosen 1 in the question doubled. As in this question, option of 1 means freshman year and option of 2 means sophomore year, this decreased average indicates that students tend to believe that they should learn computational materials science even earlier. This change in students' attitude towards the time they should learn computational skills suggests that we should incorporate computational modules even earlier in the 100-level classes.

\subsection{Perception of the importance of computation}

In this section, we show that the SIIP program raises the students awareness of the importance of computational skills. In the surveys we conduct in three different classes, MSE 201, MSE 440, and MSE 406, there are questions related to the importance of computational skills in materials science and engineering:

- "In general, do you think computational tools are important for materials science and engineering?" 


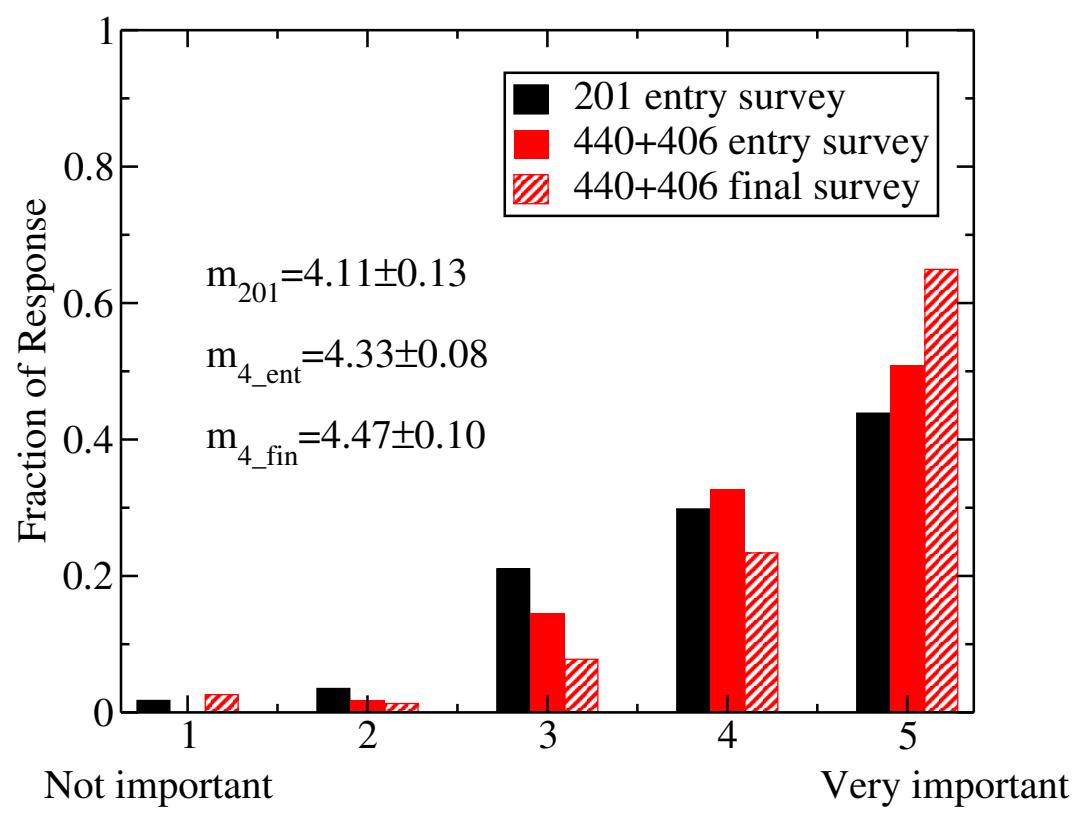

Figure 2: Students' attitude towards the importance of computational tools. The sample size is 57 for MSE 201, 110 for entry survey of MSE 440+MSE 406, and 77 for the final survey of the two senior classes. $\mathrm{m}_{\text {ent }}$ and $\mathrm{m}_{\mathrm{fin}}$ denote the mean value of the entry survey and final survey.

(MSE 201, entry survey)

- "I think computational materials science skills are important in my post-graduation career." (MSE 440, MSE 406, entry and final survey)

Figure 2 shows the results of these surveys. We aggregate responses from MSE 406 and MSE 440 to achieve a better sample size for " $400-$ level classes". The survey results indicate that the SIIP program improves student perceptions of the importance of computational skills in the materials science and engineering discipline. This can be seen in two different ways: (i) by comparing the entry-level class to the senior level classes and (ii) the entry-surveys to the final surveys. By comparing the entry survey from MSE 201 and from MSE 440+406, it can be seen that the students have better awareness of the importance of computational skills when they enter the 400-level classes, compared to when they enter the 200-level class as the average increases from $4.11 \pm 0.13$ to $4.33 \pm 0.08$. Furthermore, as students proceed and complete the 400-level classes, this average further increased from $4.33 \pm 0.08$ to $4.47 \pm 0.10$, which indicates that these 400 -level classes themselves further increase the students' awareness of their importance.

\subsection{Fulfillment of computational learning demand}

In this section, we analyze the fulfillment of students' demand of learning computational skills in their undergraduate study. The survey question we analyze is related to whether the students need more computational contents in the classes or not: 


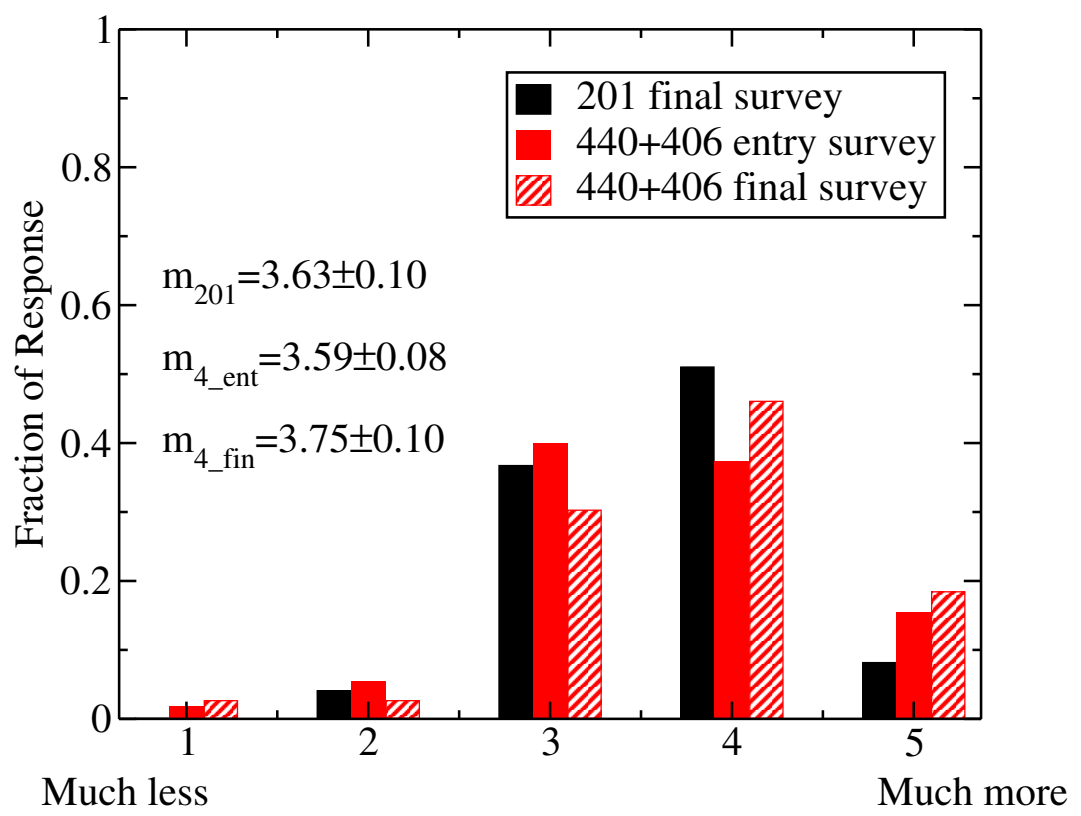

Figure 3: Students' attitude towards the amount of computational content in MSE classes. The sample size is 57 for MSE 201, 110 for entry survey of MSE $440+406$, and 76 for the final survey of the two senior classes. $\mathrm{m}_{201}$ denotes the mean value of the 201 final survey. $\mathrm{m}_{4 \text { _ent }}$ and $\mathrm{m}_{4 \text {-fin }}$ denote the mean value of the entry survey and final survey of the 400-level classes.

- "I would like to use computation in my MatSE classes ..."

(MSE 201, final survey; MSE 440 and MSE 406, entry survey and final survey)

Figure 3 shows the results of the surveys. It can be seen from the average that in all three classes, students reply that they wish to have more computational content. Interestingly, such demand increases as students complete the 400-level classes. This is another sign that the SIIP program successfully raises the students' awareness that computational skills are useful and worthy of mastering.

\section{Impact of the program within senior computational classes}

In the senior computational elective class MSE 404, we compared the survey results obtained this year with those from 2016 to analyze the impact of the SIIP sequence on students taking this class. The SIIP program was initiated two years ago, so the first group of students who experienced the program are now in their junior or senior years, at which time they may choose to take elective classes that focus completely on computational materials science. With more exposure to computational tools during their earlier curriculum, we anticipate that if the SIIP program is performing well, then these students should have better confidence in computational tools than students from previous years. Relevant computational tools are Quantum Espresso for density functional theory (DFT) calculations and LAMMPS for molecular dynamics (MD) calculations 
in MSE 404 Micro, and OOF2 for finite element method (FEM) and Thermo-Calc for phase diagram calculations in MSE 404 Macro. These were the survey questions used:

- "How confident are you in using the following computational tools?" (entry survey)

- "How confident do you feel in your ability to go out and independently use the software packages we have worked with?" (final survey)
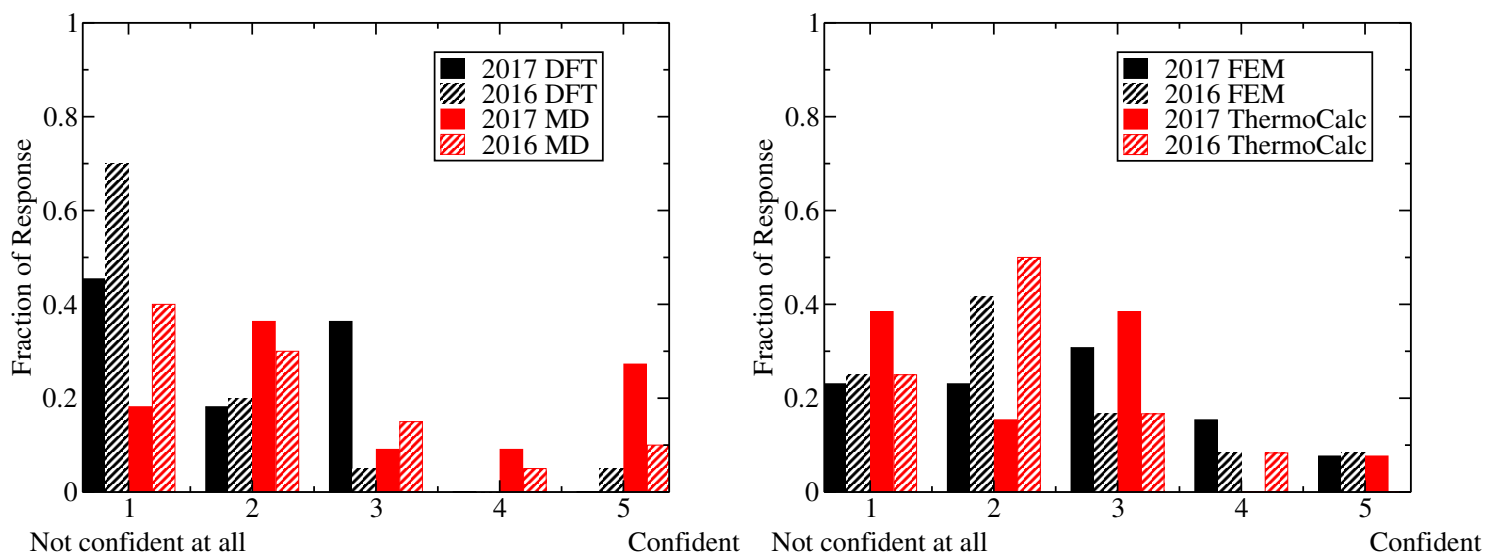

Figure 4: Students' confidence in computational tools before entering MSE 404. The sample size is 11 for Micro (2017), 13 for Macro (2017), 20 for Micro (2016), and 12 for Macro (2016).

Table 4: Table of the calculated mean value and p-value of the data for MSE 404 from year 2016 and year 2017.

\begin{tabular}{cc} 
Year/module & mean \\
\hline 2017 DFT & $1.91 \pm 0.75$ \\
2016 DFT & $1.5 \pm 0.72$ \\
2017 MD & $2.91 \pm 0.48$ \\
2016 MD & $2.15 \pm 0.49$ \\
\hline
\end{tabular}

\begin{tabular}{cc} 
Year/module & mean \\
\hline 2017 FEM & $2.62 \pm 0.41$ \\
2016 FEM & $2.33 \pm 0.49$ \\
2017 ThermoCalc & $2.23 \pm 0.56$ \\
2016 ThermoCalc & $2.08 \pm 0.61$ \\
\hline
\end{tabular}

Table 5: Table of the calculated mean value of the final survey data for MSE 404 from year 2016 and year 2017.

\begin{tabular}{lc} 
Year/module & mean \\
\hline 2017 Micro & $3.71 \pm 0.61$ \\
2016 Micro & $3.73 \pm 0.43$ \\
2017 Macro & $4.00 \pm 0.87$ \\
2016 Macro & $4.00 \pm 0.63$ \\
\hline
\end{tabular}

Figure 4 shows the histogram plot of the comparison and Table 4 shows quantitative comparison between the two data sets. We also show the students' confidence in the methods after they finish MSE 404 in Figure 5. Table 5 shows the quantitative analysis of this data. From Figure 4 and Table 4, we can see that comparing the level of confidence in the tools before entering MSE 


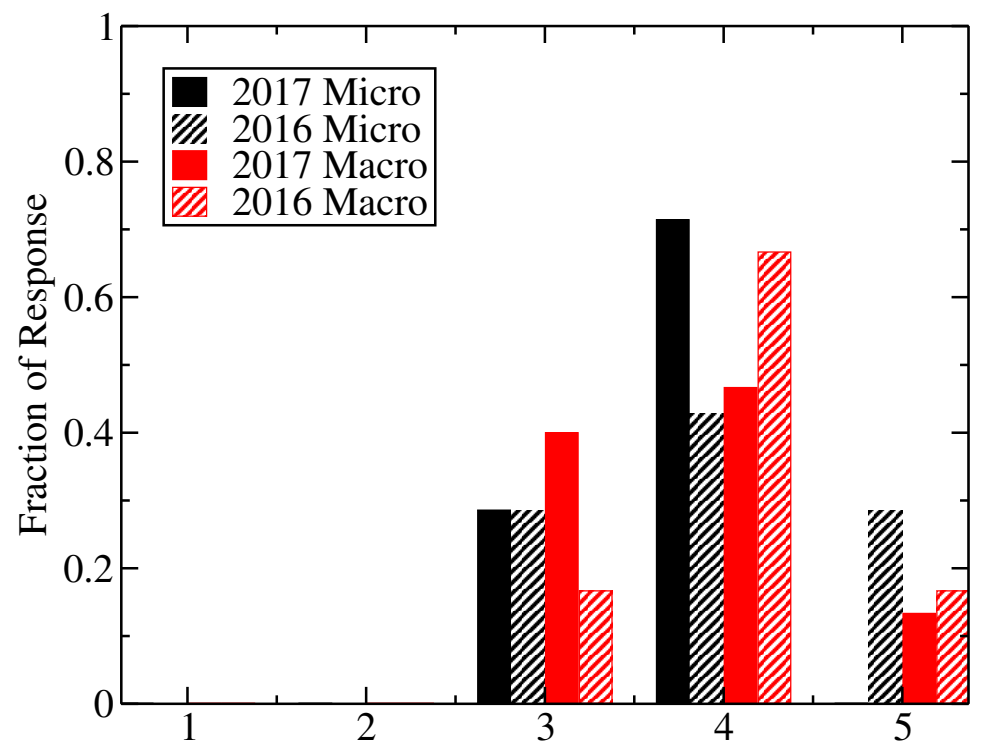

Not confident at all

Confident

Figure 5: Students' confidence in computational tools after finishing MSE 404. The sample size is 7 for Micro (2017), 7 for Macro (2017), 15 for Micro (2016), and 12 for Macro (2016).

404, students this year shows consistently more confidence compared to last year. Furthermore, as Figure 5 and Table 5 show, the level of confidence after they finish the classes does not differ much between the last two years. Due to the fact that this class is a senior level optional class, the sample size is very small thus our data has large error bars. Ways to improve feedback in order to reduce our uncertainties are discussed in section 6.

Within the senior class MSE 485, the survey questions we show are related to the students familiarity and confidence in the modules before they enter MSE 485:

- "How confident are you in using Mathematica, Matlab, programming language or simulation software to solve problems?"

- "How often do you use (simulation software/programming languages/Matlab/Mathematica) for solving problems in research or class?

The results of the survey are shown prior to and after SIIP implementation, respectively, in Fig. 6 for Spring 2015 and Fig. 7 for Fall 2017.

The last survey data of the class is from Spring 2015 because MSE 485 is only offered once every two years in the Materials Science and Engineering Department, alternating this class offering with the Physics Department. The data indicates that the computational education accrued in previous materials science classes benefits students by increasing familiarity and confidence with the simulation tools as they enter MSE 485. The average of the confidence level students have before entering the class is $3.71 \pm 0.19$. We notice that for all packages and computational tools that we have introduced in the SIIP program, the students appear to be much more confident before entering MSE 485. The average levels of familiarity are $3.00 \pm 0.25$ for all simulation packages 


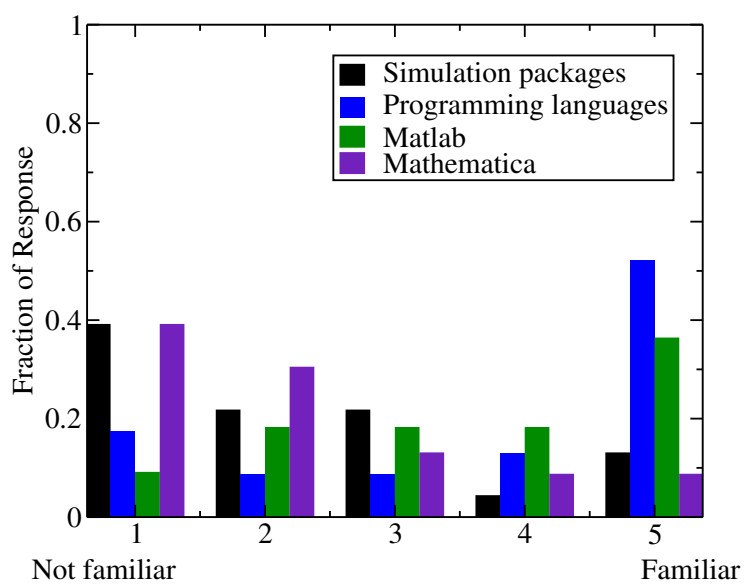

Figure 6: Students' familiarity with computational tools before entering MSE 485 in Spring 2015. The average is $2.30 \pm 0.29$ for simulation packages, $3.74 \pm$ 0.33 for programming languages, $3.55 \pm 0.30$ for Matlab, and $2.17 \pm 0.27$ for Mathematica. The sample size is 23.

(Quantum Espresso, LAMMPS, etc.), $3.85 \pm 0.20$ for programming languages and $2.76 \pm 0.22$ for Matlab. Compared with that, students familiarity to Mathematica, which is the tool that is not implemented into the curriculum, is much lower with an average of $1.85 \pm 0.15$. Comparing the results from Spring 2015 to Fall 2017, we can see a large increase of the level of confidence for simulation packages. As the simulation packages are integrated into MatSE classes exclusively through the SIIP program, it is likely that we can attribute this increase to the effectiveness of the SIIP program.

The familiarity of students with other tools do not show strong trends, as there can be other factors that may affect students' familiarity and confidence with these different methods. These factors include the fact that Matlab and programming languages are more widely used in classes involving computation, compared with simulation packages specifically for materials science, and that in MSE 485 the composition of students is more diverse and includes a substantial fraction of non-MatSE students and graduate students. Furthermore, students enrolled in MSE 485 are likely more interested in computational materials science than the overall population due to the nature of the class, and this factor can also affect students' familiarity with computational tools.

\section{Discussion}

Through a longitudinal analysis of class survey data, we have clear evidence that the SIIP program is benefiting the students by both increasing their familiarity with simulation tools and raising their awareness that computational tools are important in materials science. The survey data also provides useful information about the future work we should put into the program. Such feedback is crucial to further improve the quality of the SIIP program and of the MSE undergraduate curriculum. In particular, the aggregated survey data suggest two main directions in which to improve the computational content. First, the desire for earlier exposure to computational tools suggests that we develop new modules for the freshman classes. Second, the desire for more 

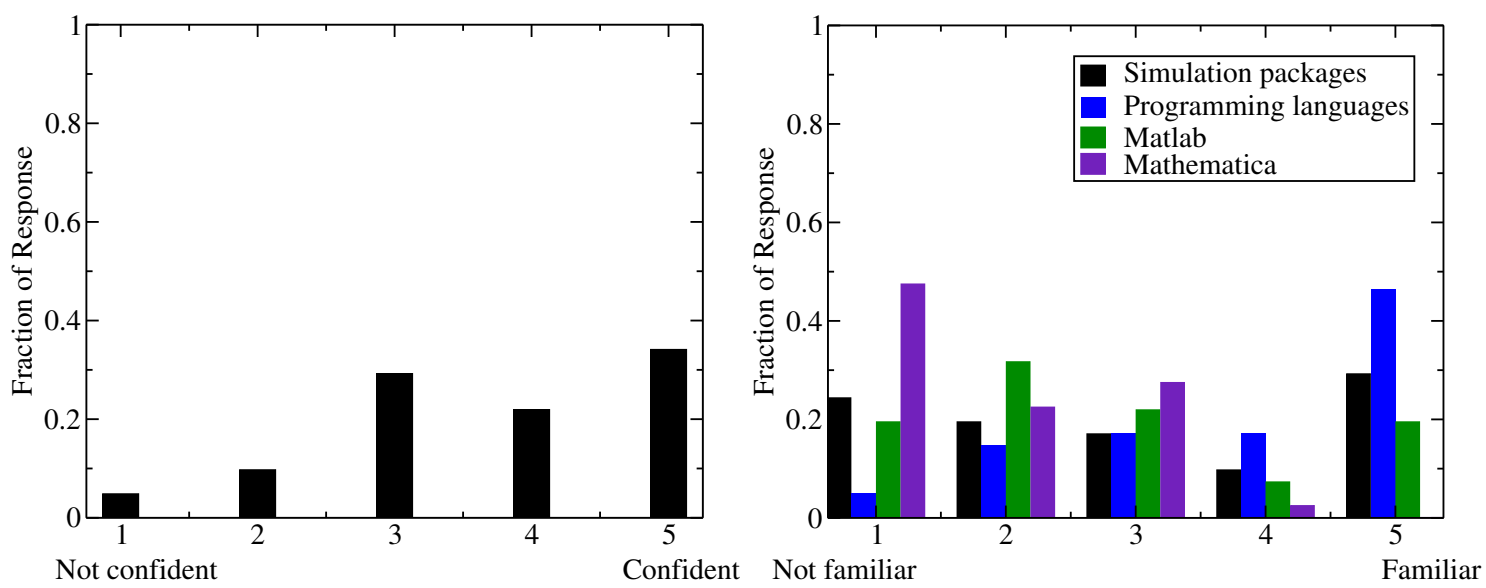

Figure 7: Students' confidence and familiarity with computational tools before entering MSE 485. The average is $3.00 \pm 0.25$ for simulation packages, $3.85 \pm$ 0.20 for programming languages, $2.76 \pm 0.22$ for Matlab, and $1.85 \pm 0.15$ for Mathematica. The sample size is 41 .

computational content in the curriculum as a whole motivates us to incorporate additional MatSE classes into the SIIP program. In addition, we also have in place a mechanism for continuous revision and improvement of the existing computational modules through an end-of-year debrief and planning session, in which the SIIP faculty and teaching assistant conduct a "post mortem" of the computational modules. By identifying aspects of the material, delivery, and student responses that were positive and successful, and those that were less so, the team identifies how and where to improve the material and its dissemination for the following year.

The student surveys provide valuable information, but their utility as an assessment instrument can benefit from further improvement. The sample sizes in the senior level computational classes are relatively small. Especially in MSE 404, the sample size is only on the order of 10-20 students each year. This results in a larger estimated standard error in the mean value and makes the data less reliable.

Furthermore, it is crucial that we develop a more complete and consistent feedback system. There are several aspects that need to be addressed in the future: First, the current surveys for different classes lack consistency. Effort should be taken to develop consistent survey questions so that we can draw more robust conclusions as students proceed through the various classes in the SIIP sequence. Second, the manner in which the surveys are administered should be standardized. At the moment, different instructors employ different methodologies, including i-clicker questions during class, integrated questions during exams, Scantron surveys in class, and online in-class feedback. It is important to look for the most efficient and effective way to conduct the surveys so that we can collect data as completely and uniformly as possible. Third, surveys should be expanded throughout all classes in the department, not just those in the SIIP program. This will be valuable especially for assessing the influence of the program on senior level students by providing a larger sample size and eliminating the self-selection sampling bias of which students choose to enroll in the computational senior electives.

Furthermore, the survey data discussed in this paper contains mostly students opinion and atti- 
tude. While this reflects the trend reasonably well, it is largely subjective. In the future, more objective data, such as information about the grades of the exams/homeworks in both the SIIP classes and computational focused classes should be included to support the survey data.

\section{Conclusion}

In this paper, we evaluate the efficacy of the implementation of computational modules into the MatSE curriculum through critical assessment of student feedback. The feedback illuminates increased student awareness of the importance of computational skills in materials science as they advance through the undergraduate program. It also suggests a demand for increased computational content and the delivery of this content earlier in the undergraduate degree program. Feedback from senior-level computational classes demonstrates that incorporation of computational content within the core curriculum measurably increases student familiarity and confidence upon entering these elective classes, providing strong support for the efficacy of the SIIP program. Finally, we observe that our feedback mechanism may be improved by enlarging sample sizes, improving consistency of questions and delivery, and supplementing with more objective measures of performance. In doing so we hope to gather improved data to further refine and improve the SIIP program to maximize student benefit.

\section{Acknowledgments}

This work was supported by the College of Engineering and the Department of Materials Science and Engineering at the University of Illinois at Urbana-Champaign as part of the Strategic Instructional Initiatives Program (SIIP), by a National Science Foundation (NSF) CAREER Award to A.L.F. (Grant No. DMR-1350008), by a NSF CAREER Award to J.A.K (Grant No. 1654182), by a NSF CAREER Award to C.L. (Grant No. 1554435), by a NSF CAREER Award to R.M. (Grant No. 1654065), and by a NSF CAREER Award to A.S. (Grant No. DMR-1555153). Any opinions, findings, and conclusions or recommendations expressed in this material are those of the authors and do not necessarily reflect the views of the NSF.

\section{References}

[1] K. Thornton, S. Nola, R. E. Garcia, M. Asta, and G. B. Olson, "Computational materials science and engineering education: A survey of trends and needs," JOM, vol. 61, no. 10, p. 12, 2009.

[2] K. Thornton and M. Asta, "Current status and outlook of computational materials science education in the US," Model. Simul. Mater. Sci. Eng., vol. 13, no. 2, p. R53, 2005.

[3] C. Vieira, A. J. Magana, R. E. García, A. Jana, and M. Krafcik, "Integrating computational science tools into a thermodynamics course,” J. Sci. Educ. Technol., pp. 1-12, 2018. 
[4] R. Mansbach et al., "Reforming an undergraduate materials science curriculum with computational modules," $J$ Mater Educ, vol. 38, pp. 161-174, 2016.

[5] A. Kononov et al., "Computational curriculum for MatSE undergraduates," in 2016 ASEE Annual Conference and Exposition, ASEE, 2017.

[6] M. Borrego and C. Henderson, "Increasing the use of evidence-based teaching in STEM higher education: A comparison of eight change strategies," J. Eng. Educ., vol. 103, no. 2, pp. 220-252, 2014.

[7] C. Henderson and M. H. Dancy, "Barriers to the use of research-based instructional strategies: The influence of both individual and situational characteristics," Phys. Rev. ST Phys. Educ. Res., vol. 3, no. 2, p. 020102, 2007.

[8] C. Henderson, A. Beach, and N. Finkelstein, "Facilitating change in undergraduate STEM instructional practices: An analytic review of the literature," J. Res. Sci. Teach., vol. 48, no. 8, pp. 952-984, 2011.

[9] C. Henderson, M. H. Dancy, and M. Niewiadomska-Bugaj, "Use of research-based instructional strategies in introductory physics: Where do faculty leave the innovation-decision process?," Phys. Rev. ST Phys. Educ. Res., vol. 8, no. 2, p. 020104, 2012.

[10] R. A. Mansbach, G. L. Herman, M. West, D. R. Trinkle, A. Ferguson, and A. Schleife, "Work in progress: Computational modules for the matse undergrad-uate curriculum," in ASEE Annual Conference \& Exposition, 2016.

[11] K. Thornton et al., "Summer school for integrated computational materials education." https://icmed. engin.umich.edu/, 2017.

[12] P. Giannozzi et al., "QUANTUM ESPRESSO: a modular and open-source software project for quantum simulations of materials," J. Phys. Condens. Matter, vol. 21, no. 39, p. 395502 (19pp), 2009.

[13] S. Plimpton, "Fast parallel algorithms for short-range molecular dynamics," J. Comput. Phys., vol. 117, no. 1, pp. 1-19, 1995.

[14] A. Stukowski, "Visualization and analysis of atomistic simulation data with OVITO-the open visualization tool," Model. Simul. Mater. Sci. Eng., vol. 18, no. 1, p. 015012, 2009.

[15] A. C. E. Reid, R. C. Lua, R. E. García, V. R. Coffman, and S. A. Langer, "Modelling microstructures with OOF2," IJMPT, vol. 35, no. 3-4, pp. 361-373, 2009.

[16] J. O. Andersson, T. Helander, L. Höglund, P. Shi, and B. Sundman, "Thermo-Calc \& DICTRA, computational tools for materials science," Calphad, vol. 26, no. 2, pp. 273-312, 2002.

[17] MATLAB, version 8.4.0 (R2014b). Natick, Massachusetts: The MathWorks Inc., 2014. 